\title{
Dissecting transcription regulatory pathways through a new bacterial one-hybrid reporter system
}

\author{
Manman Guo, Hui Feng, Jun Zhang, Wenqin Wang, Yi Wang, Yuqing Li, Chunhui Gao, \\ Huanchun Chen, Ying Feng, and Zheng-Guo $\mathrm{He}^{1}$ \\ National Key Laboratory of Agricultural Microbiology, Center for Proteomics Research, College of Life Science and Technology, \\ Huazhong Agricultural University, Wuhan 430070, China
}

\begin{abstract}
Sequence-specific DNA-binding transcription factors have widespread biological significance in the regulation of gene expression. However, in lower prokaryotes and eukaryotic metazoans, it is usually difficult to find transcription regulatory factors that recognize specific target promoters. To address this, we have developed in this study a new bacterial one-hybrid reporter vector system that provides a convenient and rapid strategy to determine the specific interaction between target DNA sequences and their transcription factors. Using this system, we have successfully determined the DNA-binding specificity of the transcription regulator Rv3133c to a previously reported promoter region of the gene Rv2031 in Mycobacterium tuberculosis. In addition, we have tested more than 20 promoter regions of M. tuberculosis genes using this approach to determine if they interact with $\sim 150$ putative regulatory proteins. A variety of transcription factors are found to participate in the regulation of stress response and fatty acid metabolism, both of which comprise the core of in vivo-induced genes when $M$. tuberculosis invades macrophages. Interestingly, among the many new discovered potential transcription factors, the WhiB-like transcriptional factor WhiB3 was identified for the first time to bind with the promoter sequences of most in vivo-induced genes. Therefore, this study offers important data in the dissection of the transcription regulations in M. tuberculosis, and the strategy should be applicable in the study of DNA-binding factors in a wide range of biological organisms.
\end{abstract}

[Supplemental material is available online at www.genome.org.]

Specific DNA-protein interactions are important in numerous cellular processes, such as transcription regulation, DNA replication, and repair. However, although it is easy to computationally predict these cis or in cis factors (Berman et al. 2002), it is usually quite difficult in practice to find the specific target promoter recognized by a transcription factor of interest. This is particularly true in the case of disease-causing pathogens, where the role of transcription factors and DNA-protein interactions in imparting pathogenicity remains poorly understood. With the recent sequencing of many microorganisms, including many bacterial pathogens, attention has shifted toward the determination of the regulatory mechanism underlying gene expression patterns. The purpose of this is to better understand host-pathogen interactions and diseases.

One organism of interest in this context is Mycobacterium tuberculosis, the causative microbe for tuberculosis (TB). Mycobacterial infection has been a major cause of death throughout human history and still results in the death of about two million people globally each year. This enduring pathogenicity suggests that this Mycobacterium may use unique pathogenic mechanisms during its infection process. $M$. tuberculosis confronts a more hostile environment during infection, including restricted access to nutrients and reduced oxygen tension (James et al. 1995; Schaible et al. 1998; Via et al. 1998). Indeed, oxygen tension is one factor frequently associated with the establishment and maintenance of latent TB (Wayne and Sohaskey 2001). Therefore, M. tuberculosis must possess genetic mechanisms to facilitate integrated responses to multiple stresses encountered within the phagosome, and also

\footnotetext{
${ }^{1}$ Corresponding author.
}

E-mail hezhengguo@hotmail.com; fax 86-27-87280670.

Article published online before print. Article and publication date are at http://www.genome.org/cgi/doi/10.1101/gr.086595.108. to trigger some yet-to-be-identified switches during the infection process (Talaat et al. 2004; Rengarajan et al. 2005; Rohde et al. 2007).

When an intracellular pathogen such as M. tuberculosis enters activated macrophages, it confronts a particularly stressful environment, so it must possess the means of conveying information to the transcriptional apparatus to promote its environmental adaptation. One initial obstacle concerns the acquisition of essential nutrients. Isocitrate lyase (ICL), an enzyme essential for the metabolism of fatty acids, also appears to be important for the survival of the pathogen in the lungs of mice during the persistent phase of infection (McKinney et al. 2000; Muñoz-Elías and McKinney 2005). The induction of genes involved in the $\beta$-oxidation of fatty acids during $M$. tuberculosis persistent infections suggests that fatty acids might be a major source of carbon and energy in chronically infected lung tissue (Sassetti and Rubin 2003). In addition to this, M. tuberculosis appears to have sophisticated genetic mechanisms to recognize stressful environment signals, such as $\mathrm{O}_{2}, \mathrm{NO}$, and fluctuations in the intracellular redox state (Singh et al. 2007). The genes involved in fatty acid metabolism and stress responses have been suggested to comprise the core of in vivo-regulated genes (Talaat et al. 2004). However, little is known regarding the molecular mechanisms behind the sensing of extracellular signals and nutrient stress by $M$. tuberculosis in the course of metabolic adaptation.

Sequence-specific DNA-binding transcription factors have widespread biological significance in regulating gene expression. Several reports have described the expression profiles of M. tuberculosis genes during infection using DNA microarrays, quantitative real-time RT-PCR, and the mouse model of tuberculosis (Sassetti and Rubin 2003; Talaat et al. 2004; Rengarajan et al. 2005; Rohde et al. 2007). The genome of M. tuberculosis encodes 13 RNA 
polymerase $\sigma$-factors, as well as more than 140 putative regulatory proteins, which suggest an important role of transcriptional regulation in the life cycle of M. tuberculosis (Cole et al. 1998; Timm et al. 2003). Of these, Rv3133c is one of the very few M. tuberculosis transcription factors for which a role has been characterized (Park et al. 2003). Rv3133c was shown to be required for the hypoxic induction of the alpha-crystallin encoded by Rv2031 (acr) (Sherman et al. 2001). The Acr is a dominant antigen and was shown to be recognized by most TB patient sera (Lee et al. 1992; Verbon et al. 1992). Several studies demonstrated that the expression of Rv2031 was dramatically and rapidly increased under hypoxic conditions (Yuan et al. 1998; Florczyk et al. 2001; Sherman et al. 2001). Park et al. (2003) found that Rv3133c bound with motif sequences upstream of the acr coding region, and this binding was necessary for hypoxic gene induction. Recently, Boon et al. (2002) demonstrated that the long-term hypoxic survival of $M$. bovis BCG required Rv3133c (named DosR for dormancy survival regulator). Although recent studies have confirmed the importance of gene regulatory networks in MTB pathogenesis (Graham and Clark-Curtiss 1999; Chen et al. 2000; McKinney et al. 2000; Zahrt and Deretic 2000; Manganelli et al. 2001; Wilkinson et al. 2001), the target genes and the cellular processes controlled by these transcription factors are largely unknown.

In this study, we have developed a new bacterial one-hybrid system in which the reporter vector has been integrated with a rapid and simple cloning strategy for polymerase chain reaction (PCR) products. In addition, it is compatible with many commercially available pTRG cDNA libraries. Using this new system, we have successfully isolated a number of new transcriptional factors involved in the induction of in vivo-regulated genes in the pathogen M. tuberculosis H37Rv. A WhiB-like transcriptional factor, WhiB3 (Rv3416), that extensively regulates fatty acid metabolism and stress responses by binding with the promoter sequences of many of the genes involved is likewise detected.

\section{Results}

\section{A new bacterial one-hybrid reporter vector containing the $\mathrm{Xcml}$ cassette DNA}

The BacterioMatch II two-hybrid system (Stratagene) is an efficient method to detect protein-protein interactions in vivo using the pBT bait plasmid and a pTRG library. The new bacterial one-hybrid (B1H) reporter vector $\mathrm{pBX} \mathrm{cmT}$ is derived from the vector $\mathrm{pBT}$ and contains three major components including the reporter cassette, the XcmI cassette, and the mediator DNA fragment (Supplemental Fig. 1). The HIS3-aadA system has been shown to be a successful reporter system for bacterial two-hybrid screening. These two genes, HIS3 and aadA, were also used as the reporter cassette in the one-hybrid system. The $\mathrm{XcmI}$ cassette, containing two XcmI sites in both of its termini, was constructed and integrated into the $\mathrm{pBXcmT}$. When digested with $\mathrm{XcmI}$, the recombinant plasmid $\mathrm{pBX} \mathrm{cmT}$ results in a vector with a single deoxythymidine (dT) overhanging at its $3^{\prime}$-end, which can be used for the rapid cloning of PCR products. To restrain the selfactivation and reduce the screening background, a mediator DNA fragment was successfully cloned into the right upstream of the XcmI cassette (Supplemental Fig. 1).

The reporter plasmid $\mathrm{pBXcmT}$ is a derivative vector of the bacterial two-hybrid pBT bait plasmid. Thus, the new one-hybrid vector is compatible with both the BacterioMatch II validation reporter strain and the commercially available pTRG cDNA libraries (Stratagene). When a target protein expressed in pTRG interacts with the bait DNA in the reporter vector of pBXcmT, it stabilizes the binding of RNA polymerase at the weak promoter and activates the transcription of the HIS 3 and aadA reporter genes in the presence of the compound 3-amino-1,2,4-triazole (3-AT) (Fig. 1).

\section{Specific DNA-protein interactions between the transcription factor and the promoter of M. tuberculosis as determined by the new reporter system}

Rv3133c has been previously shown to bind to motif sequences upstream of the acr (Rv2031) coding region and its C-terminal HTH DNA-binding domain is essential for the binding (Fig. 2A). The acr promoter contains high-affinity DevR-binding sequences, and the mutation of these sequences abolished the DevR binding and resulted in dramatically reduced levels of expression (Fig. 2B; Park et al. 2003). The sequence-specific interaction between Rv3133c and the promoter of Rv2031 was used to test the new B1H system.

The promoter containing the DNA fragment was amplified using specific primers (Supplemental Table 1) and was inserted directly into the reporter vector $\mathrm{pBXcmT}$. The wild-type and mutant Rv3133c gene was cloned into the pTRG. As shown in Figure 2C, the strain with $\mathrm{pR} 2031 \mathrm{wt} / \mathrm{pRv} 3133 \mathrm{c}$ grew very well, while that with pR2031/pRv3133 $\Delta$ C failed to grow, indicating that the ability to activate reporter gene expression is lost when the C-terminal DNA-binding domain is deleted. In addition, when essential promoter bases of Rv2031 were mutated, the growth of the mutant strain was significantly reduced on the selective medium; the selfactivation control strain did not grow under the same conditions (Fig. 2C).

To further examine the correlation between growth of the reporter strain and the specific DNA-protein interactions, we conducted surface plasmon resonance (SPR) assays that compared the in vitro DNA-binding activity of wild-type and mutant Rv3133c using purified proteins. As shown in Figure 2, the wildtype Rv3133c protein had the best affinity for the Rv2031 promoter DNA immobilized on the chips, while Rv3133 $\Delta$ C lost almost all the affinity for the DNA (Fig. 2D). In contrast, when the promoter sequence was mutated, the response unit (RU) bound by the Rv3133c protein had an obviously lower affinity than the wild-type promoter (Fig. 2D). Thus, these results are perfectly consistent with those from our B1H assay. The wild-type Rv3133c was best at activating transcription by the Rv2031 promoter, while

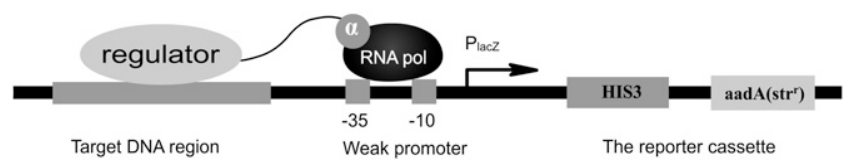

Figure 1. Schematic of the bacterial one-hybrid reporter system. In the new bacterial one-hybrid system, a selected promoter sequence or the other genomic DNA, the bait DNA, was cloned into the reporter vector of $\mathrm{pBXcmT}$ containing the selectable genes HIS3 and aadA. A given transcription factor or a library was expressed as a fusion to the alpha subunit of the RNA polymerase in the vector of pTRG. The reporter genes would be activated in case of interaction of the promoter region with a given regulator protein expressed in PTRG when a pair of recombinant vectors is cotransformed into the host strain. If the regulator protein interacts with the target DNA, it recruits and stabilizes the binding of RNA polymerase at the weak promoter and thus activates the transcription of the HIS 3 reporter gene in the presence of the compound 3-amino-1,2,4-triazole (3-AT). A second reporter gene aadA encoding a protein that confers streptomycin resistance provides an additional mechanism to validate the DNA-protein interaction.

\section{Genome Research www.genome.org}


A

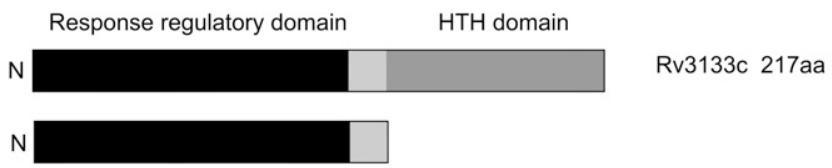

B

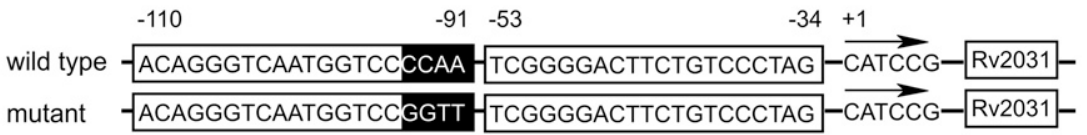

C

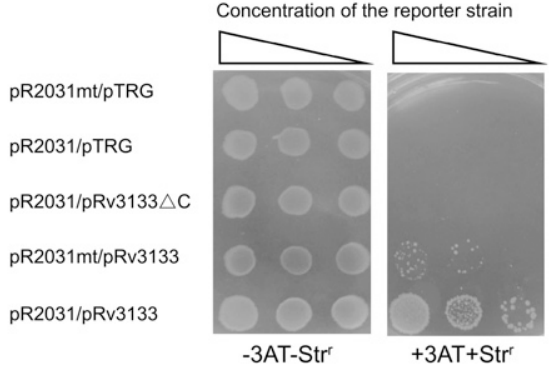

D
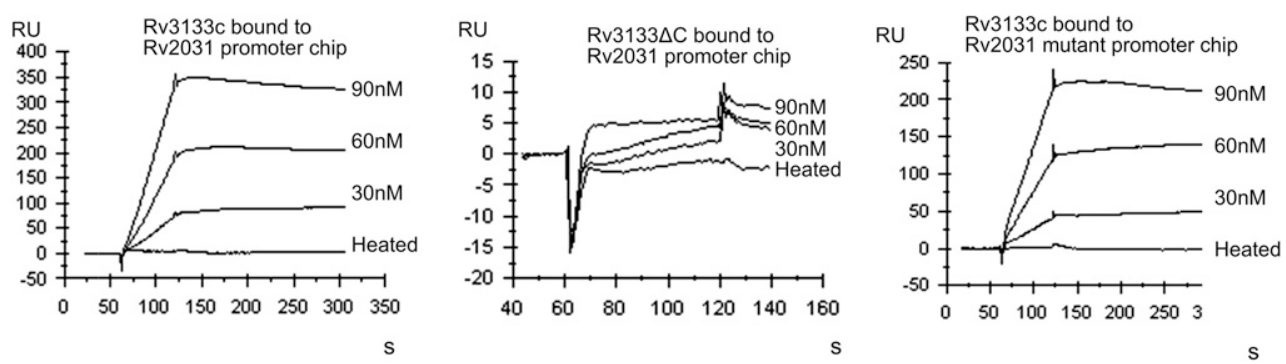

Figure 2. DNA-binding specificity examined by the bacterial one-hybrid reporter system. (A) A 143 amino acid mutant protein of Rv3133c (DevR) was produced by deleting its C-terminal domain and used for the DNA-binding specificity assay. (B) A mutant promoter of Rv2031 was constructed by mutating its CCAA as GGTT, as shown in black background. (C) The bacterial one-hybrid assays. The promoters were cloned into pBXcmT. Rv3133c and its C-terminal deleted mutant, Rv3133 $\Delta$ C, were cloned into PTRG vectors, respectively. The E. coli XL1-Blue MRF' Kan strain was used for the routine propagation of all $\mathrm{pBX} \mathrm{cmT}$ and $\mathrm{pTRG}$ recombinant plasmids. A pair of $\mathrm{pBX} \mathrm{cmT} / \mathrm{pTRG}$ plasmids was co-transformed into the reporter strain and then its growth was tested together with the self-activation controls on a selective medium containing 3-AT, $\mathrm{Kan}^{r}$, Str ${ }^{r}$, and Chl'. Positive growth cotransformants were selected on the selective screening medium plate. (D) SPR assays for protein-DNA interactions. Both Rv3133C and Rv3133 $\Delta$ C were expressed in E. coli and purified. Biotin-labeled promoter DNA was immobilized on the SA chips (BIAcore). Following a period of stabilization, Rv3133c or Rv3133 $\Delta \mathrm{C}$ protein was passed over the chip. Each analysis was performed in triplicate. An overlay plot was produced for depicting the interaction between Rv3133c or Rv3133 C with different concentrations of promoter DNA. RU, response units; s, seconds.

Rv3133 $\Delta$ C lost most of its activation function, and the affinity of the mutant Rv2031 promoter for the Rv3133c protein significantly reduced (Fig. 2D).

Specific interaction between a transcription factor and its target promoter DNA could therefore be successfully detected using the bacterial one-hybrid system described in this study.

\section{Detection of new transcription regulators for the expression of Rv2031 and Rv3874/3875}

To further test the new B1H system and to explore potential new transcription regulators, the promoters of Rv2031 and Rv3874/3875 were used to screen an $M$. tuberculosis subgenomic library in which almost all transcription factor genes were cloned using specific PCR primers. The potential regulators that could interact with the promoters were isolated from the library of M. tuberculosis H37Rv.

As shown in Figure 3A, six potential regulatory genes for Rv2031 were isolated. Rv3133c was again characterized as the transcription factor under the screening condition used. The reg- ulators included a hypothetical Rv3678c protein, an RNA polymerase Rv0445c sigma factor (SigK), two WhiB-like transcriptional regulatory proteins, Rv3416 and Rv3681c, and the probable transcriptional regulatory Rv2034 proteins. To confirm these findings, we examined the interactions between the Rv2031 promoter and these new proteins in vitro using SPR assays. As shown in Figure 3B, all four probable regulators, Rv3678, Rv3681, Rv3416, and Rv2034, demonstrated obvious affinities to the promoter DNA and produced significant binding signals, which disappeared after heat inactivation.

ESAT-6 and CFP-10, which were encoded by Rv3874 and Rv3875, respectively, are two major secretion antigens of $M$. tuberculosis required to control host-cell response to infection (Champion et al. 2006; Derrick and Morris 2007). Thus, the expression of these two proteins would be expected to be tightly regulated during the infection. Using the new B1H system, when the promoter of Rv3874/3875 was cloned and the library screened, three regulator factors were isolated, including two RNA polymerase sigma factors, Rv2710 (SigB) and Rv3414 (SigD), and 
A

Transcription regulatory factors
Rv0445c(SigK), Rv2034, Rv3133c(DevR),
Rv3416 (WhiB3), Rv3678, Rv3681c(WhiB4)

Rv2021, Rv2710(SigB), Rv3414(SigD)

B
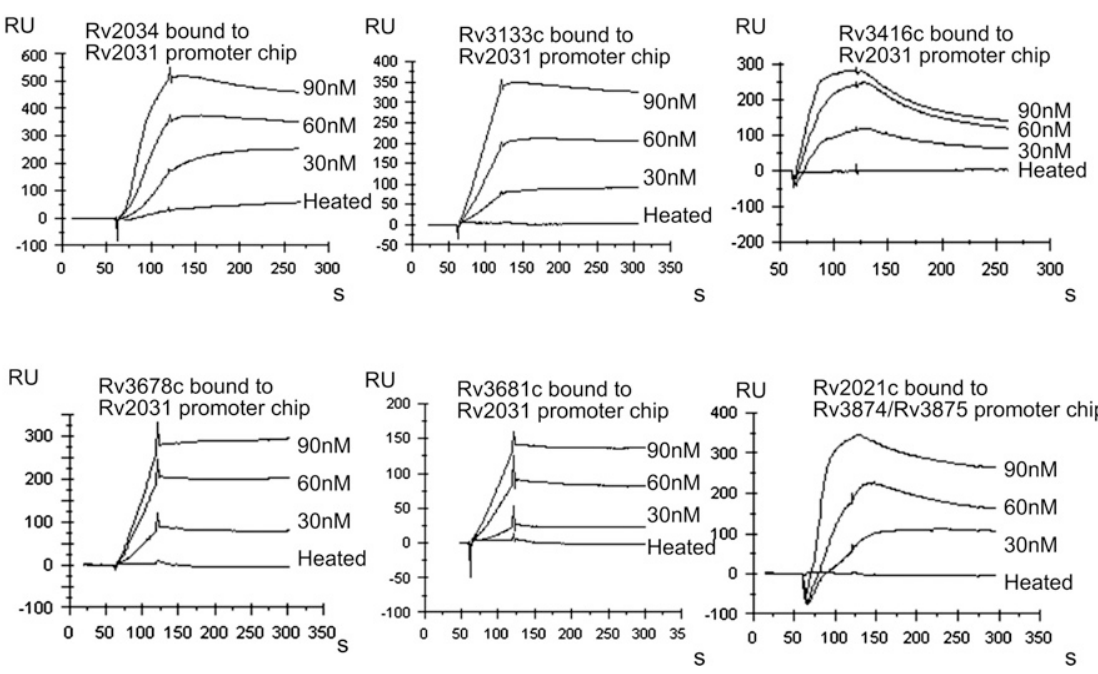

Figure 3. Characterization of novel transcription factors by the one-hybrid system in combination with SPR assays. (A) Detection of novel transcription factors by the bacterial one-hybrid system. The promoters of Rv2031 and Rv3874/3875 were cloned in pBXcmT and were used to screen the M. tuberculosis subgenomic library according to the description in Materials and Methods. The regulators that interact with the promoters were isolated from the library and are listed on the left. The structure of the operon of Rv2031 or the Rv3874/3875 is listed on the right. (B) SPR assays for the interaction between the new transcription factors and the promoter. Several new transcription factors of Rv2021, Rv2034, Rv3416, Rv3678, and Rv3681 were expressed and purified. The SPR assaying procedure followed the previous description. An overlay plot was produced for depicting the interaction between the promoter DNA with the different concentrations of transcription factor $(30 \mathrm{nM}, 60 \mathrm{nM}$, and $90 \mathrm{nM})$. RU, response units; s, seconds.

a probable transcriptional regulatory protein Rv2021 (Fig. 3A). The Rv3874/3875 promoter binding activity of Rv2021 was also confirmed by the in vitro SPR assay (Fig. 3B).

\section{Transcriptional regulation of the stress response genes in M. tuberculosis}

Stress response genes are in vivo-induced genes involved in sensing signals such as oxygen, nitric oxide, and nutrient depletion during infection (Talaat et al. 2004). However, the transcription factors essential for regulating the expression of these genes have not yet been characterized. When the promoter sequences of eight in vivoinduced stress response genes reported in several previous studies (Talaat et al. 2004; Rengarajan et al. 2005; Rohde et al. 2007) were used as baits to screen the library (Supplemental Table 2), a twocomponent regulator, RegX3 (Rv0491), and a WhiB-like transcriptional factor, WhiB3 (Rv3416), were detected that bound with most of the regulating sequences of the stress response genes (Table 1; Supplemental Fig. 2). Recently, Singh et al. (2007) suggested that WhiB3 responded to the dormancy signals, $\mathrm{NO}$ and $\mathrm{O}_{2}$, through its iron-sulfur cluster. The RegX3 has also been reported to participate in the adaptation to the host environment (Parish et al. 2003).

The results from the current study offer further evidence for the involvement of these two genes in the regulation on the ex- pression of stress response genes in $M$. tuberculosis. DevR (Rv3133c) was bound to the regulating sequence of the Rv3132Rv3134c gene cluster, including Rv3133c itself. Thus, our results suggest that the Rv3133c gene can self-regulate its own expression, which is consistent with a previous report that used an in vitro assay (Park et al. 2003). Rv3414 (SigD) binding with the promoter of the Rv3417-Rv3418 gene cluster encoding GroEL1 is consistent with a previous result from the whole genomic microarray analysis, in which both Rv3417 and Rv3418 were found to have a twofold lower expression in the SigD M. tuberculosis mutant strain (Raman et al. 2004).

More than 40 previously unknown interactions of regulators with specific promoters were detected by using the new B1H reporter system (Table 1; Supplemental Fig. 2). These findings provide important information to understand the mechanism of stress responses in $M$. $t u$ berculosis.

\section{Transcriptional switching factors for the fatty acid metabolism in M. tuberculosis}

Fatty acids have been suggested as a major source of carbon and energy in a chronically infected lung tissue (McKinney et al. 2000). Although fatty acid metabolism genes form part of the core of in vivoinduced genes during infection (Talaat et al. 2004), the essential metabolic switching factors that regulate their expressions are not known. The regulating sequences from 11 in vivo-induced fatty acid metabolism genes or gene clusters reported in several previous studies (Talaat et al. 2004; Rengarajan et al. 2005; Rohde et al. 2007) were used to screen the library (Supplemental Table 2). As shown in Table 2 and Supplemental Figure 3, an oxygen stress regulator OxyS, encoded by Rv0117, bound the regulating sequence of the Rv2903-Rv2939 gene cluster including FadD26 (Rv2930), and that of the Rv3537-Rv3538 gene cluster including UfaA2. DevR (Rv3133c) was involved in the expression of the FadD9, Icl/FadB2, and FadE26/27 genes or gene clusters; one of which, Icl (Rv0467), is a crucial up-regulated enzyme of fatty acid metabolism in vivo in M. tuberculosis (McKinney et al. 2000). On the other hand, WhiB3 was detected to extensively bind with the regulating sequences of almost all fatty acid metabolism genes in this study, except for Rv1094 (DesA2). To further map the binding region of WhiB3 within the regulatory sequence of the $\mathrm{icl}$ gene, we conducted an SPR assay using two truncated DNA fragments (Supplemental Fig. 4A). An $\sim 60$ bp region was shown to contain the potential target site due to a significantly reduced binding signal of WhiB3 detected on the shorter fragment, which had lost the 60-bp region (Supplemental Fig. 4B). A further BLAST assay to search for the potential binding site defined a relatively flexible DNA-binding motif, TNGCGNTNNCGC for WhiB3 (Supplemental Fig. 4C), within the regulatory sequences of the target

\section{Genome Research www.genome.org}


Table 1. Regulators for stress response genes detected by the new one-hybrid system

\begin{tabular}{|c|c|c|}
\hline Regulators & $\begin{array}{l}\text { Target gene or } \\
\text { gene cluster }\end{array}$ & $\begin{array}{l}\text { Proteins encoded by } \\
\text { target gene cluster }\end{array}$ \\
\hline Rv0117(Oxys) & Rv1907-1912 & KatG, FurA \\
\hline Rv0260 & Rv1460-1466 & \\
\hline \multirow[t]{2}{*}{ Rv0445c(SigK) } & $\begin{array}{l}\text { Rv0440 } \\
\text { Rv1460-1466 }\end{array}$ & GroEL2 \\
\hline & Rv3197A & WhiB7 \\
\hline \multirow[t]{5}{*}{ Rv0491(RegX3) } & Rv350-353 & DnaK, GrpE \\
\hline & Rv3132-3134 & DosR \\
\hline & Rv3249-3252 & RubB, RubA \\
\hline & Rv3417-3418 & GroEL1 \\
\hline & Rv3197A & WhiB7 \\
\hline \multirow[t]{2}{*}{ Rv0818 } & Rv1460-1466 & \\
\hline & Rv3249-3252 & RubB, RubA \\
\hline \multirow[t]{2}{*}{ Rv0981(MprA) } & Rv350-353 & DnaK, GrpE \\
\hline & Rv3417-3418 & GroEL1 \\
\hline Rv1221(SigE) & Rv350-353 & DnaK, GrpE \\
\hline \multirow[t]{2}{*}{ Rv1359 } & Rv1460-1466 & \\
\hline & Rv3249-3252 & RubB, RubA \\
\hline Rv1931c & Rv1460-1466 & \\
\hline \multirow[t]{2}{*}{ Rv1956 } & Rv3197A & WhiB7 \\
\hline & Rv3249-3252 & RubB, RubA \\
\hline Rv1990c & Rv0440 & GroEL2 \\
\hline Rv2017 & Rv3132-3134 & DosR \\
\hline \multirow[t]{2}{*}{ Rv2021c } & Rv0440 & GroEL2 \\
\hline & Rv3417-3418 & GroEL1 \\
\hline \multirow[t]{4}{*}{ Rv2034 } & Rv350-353 & DnaK, GrpE \\
\hline & Rv0440 & GroEL2 \\
\hline & Rv1907-1912 & KatG, FurA \\
\hline & Rv3197A & WhiB7 \\
\hline Rv2669 & Rv3132-3134 & DosR \\
\hline Rv2745c & Rv3417-3418 & GroEL1 \\
\hline \multirow{2}{*}{ Rv3133c } & Rv350-353 & DnaK, GrpE \\
\hline & Rv3132-3134 & DosR \\
\hline Rv3414(SigD) & Rv3417-3418 & GroEL1 \\
\hline \multirow[t]{5}{*}{ Rv3416(WhiB3) } & Rv350-353 & DnaK, GrpE \\
\hline & Rv1460-1466 & \\
\hline & Rv1907-1912 & KatG, FurA \\
\hline & Rv3197A & WhiB7 \\
\hline & Rv3417-3418 & GroEL1 \\
\hline Rv3557c & Rv1460-1466 & \\
\hline Rv3678c & Rv3417-3418 & GroEL1 \\
\hline Rv3681(WhiB4) & Rv3249-3252 & RubB, RubA \\
\hline Rv3692 & Rv3132-3134 & DosR \\
\hline Rv3833 & Rv3132-3134 & DosR \\
\hline
\end{tabular}

genes detected by the bacterial one-hybrid system (Supplemental Table 3). Since WhiB3 holds an iron-sulfur (Fe-S) cluster to respond to the stress signals $\mathrm{NO}$ and $\mathrm{O}_{2}$, Singh et al. have suggested that WhiB3 might be a metabolic switching and intracellular redox sensor in M. tuberculosis (Singh et al. 2007). Our finding that WhiB3 could extensively regulate fatty acid metabolism and stress responses by binding directly with their promoter sequence supported and extended this conclusion.

As shown in Table 2, $\sim 60$ new interactions of regulators with specific promoters involved in the regulation of fatty acid metabolism were detected using the new $\mathrm{B} 1 \mathrm{H}$ reporter system (Table 2). These findings suggest that the fatty acid metabolism in M. tuberculosis might be tightly regulated through a yet-to-be-identified mechanism.

\section{Discussion}

A lack of data on the DNA-binding specificities of most transcription factors has been a major obstacle in understanding gene regulatory networks (Bulyk 2005). The limitations of current methods, including SELEX (Roulet et al. 2002) and genome-wide chromatin immunoprecipitation (ChIP-chip) (Lee et al. 2002) and DNA immunoprecipitation with microarray detection (DIP-chip) (Liu et al. 2005), are their reliance on specific antibodies and their requirement of expensive facilities and expertise for further data analysis. Some in vitro technologies, such as EMSA, require purified active proteins and radioactive labeling of the target DNA. In contrast, bacterial one-hybrid systems not only use in vivo selection but also offer a low-technology alternative to microarraybased technologies. Using this kind of technology, Meng et al. (2005) examined successfully the DNA-binding specificity of seven previously characterized transcription factors and one novel transcription factor from Drosophila melanogaster. However, existing one-hybrid systems usually require de novo library construction, and thus the data from different labs are not readily comparable.

In the bacterial one-hybrid system presented here, we expressed the DNA-binding domain of a given DNA-binding protein, such as a transcription factor, or a cDNA library as a fusion to the alpha subunit of the RNA polymerase in the PTRG vector. A PCR product of an interesting target DNA or a library of genomic DNA can be conveniently cloned into the reporter vector pBXcmT containing the selectable genes HIS3 and aadA. A mediator sequence factor integrated into the upstream of the reporter cassette significantly reduces the self-activation background (data not shown). An obvious advantage of this new system is that an easy cloning strategy was integrated into the reporter vector, which is very convenient for the cloning of short target DNA PCR products. In contrast to other bacterial one-hybrid systems, the reporter vector in our system is compatible with commercial pTRG libraries such as humans, rice, and yeast, and can be partnered to identify specific DNA-protein interactions.

The use of a rapid PCR product cloning strategy for the DNA target factor in a reporter vector and its compatibility with many commercial pTRG cDNA libraries represent two important advantages of our method over existing bacterial one-hybrid selection systems. More importantly, the system works very well in detecting the specific DNA-protein interactions of the M. tuberculosis transcription factors. Either the transcription factor, or its DNA target sites, could be mutated and the specificities of the interactions were interrupted (Fig. 2). These results, along with those from a previous study (Meng et al. 2005) in which the DNAbinding specificities of eukaryotic transcriptional factors were successfully examined, show that the bacterial one-hybrid system can be utilized to characterize DNA-binding specificity in bacteria and eukaryote. Furthermore, the bacterial system could also be used to successfully detect the DNA-binding specificity of eukaryote-like archaeal proteins (data not shown).

The bacterial one-hybrid system may be limited in its scalability and may also not be suitable for determining specificity under some conditions, such as when the DNA binding proteins are modified post-translationally and only then bind to the target sequences. In addition, it is a probably common problem for bacterial one-hybrid systems to produce potential false positive/ negative results. Therefore, controls that test known transcription factor/promoter interactions should be run to determine the most suitable screening conditions. Validation of the data obtained from library screens using different methods such as SPR, EMSA, or whole genomic microarray analysis should also be carried out. Comprehensive evaluation of data derived from multiple experiments should be considered before conclusions are drawn. 
Table 2. Regulators for fatty acid metabolism genes detected by the new one-hybrid system

\begin{tabular}{|c|c|c|}
\hline Regulators & $\begin{array}{l}\text { Target gene or } \\
\text { gene cluster }\end{array}$ & $\begin{array}{l}\text { Proteins encoded by } \\
\text { target gene cluster }\end{array}$ \\
\hline Rv0117(OxyS) & $\begin{array}{l}\text { Rv2930-2939 } \\
\text { Rv3537-3538 }\end{array}$ & $\begin{array}{l}\text { FadD26 } \\
\text { UfaA2 }\end{array}$ \\
\hline Rv0445c(SigK) & $\begin{array}{l}\text { Rv3543-3545 } \\
\text { Rv3550-3552 } \\
\text { Rv3504-3505 } \\
\text { Rv2930-2939 } \\
\text { Rv0244 } \\
\text { Rv0551 }\end{array}$ & $\begin{array}{l}\text { FadE28, FadE29 } \\
\text { EchA20 } \\
\text { FadE26, FadE27 } \\
\text { FadD26 } \\
\text { FadE5 } \\
\text { Fad8 }\end{array}$ \\
\hline Rv0491(RegX3) & Rv3537-3538 & UfaA2 \\
\hline Rv0818 & Rv3543-3545 & FadE28, FadE29 \\
\hline Rv0823c & $\begin{array}{l}\text { Rv3550-3552 } \\
\text { Rv0551 }\end{array}$ & $\begin{array}{l}\text { EchA20 } \\
\text { Fad8 }\end{array}$ \\
\hline Rv1359 & $\begin{array}{l}\text { Rv2930-2939 } \\
\text { Rv3504-3505 }\end{array}$ & $\begin{array}{l}\text { FadD26 } \\
\text { FadE26, FadE27 }\end{array}$ \\
\hline Rv1931c & Rv3550-3552 & EchA20 \\
\hline Rv1956 & $\begin{array}{l}\text { Rv3550-3552 } \\
\text { Rv3543-3545 } \\
\text { Rv3537-3538 } \\
\text { Rv2930-2939 }\end{array}$ & $\begin{array}{l}\text { EchA20 } \\
\text { FadE28, FadE29 } \\
\text { UfaA2 } \\
\text { FadD26 }\end{array}$ \\
\hline Rv2017 & $\begin{array}{l}\text { Rv3543-3545 } \\
\text { Rv0467-0468 }\end{array}$ & $\begin{array}{l}\text { FadE28, FadE29 } \\
\text { Icl, FadB2 }\end{array}$ \\
\hline Rv2021c & $\begin{array}{l}\text { Rv0551 } \\
\text { Rv2590 } \\
\text { Rv3537-3538 }\end{array}$ & $\begin{array}{l}\text { Fad8 } \\
\text { FadD9 } \\
\text { UfaA2 }\end{array}$ \\
\hline Rv2034 & $\begin{array}{l}\text { Rv3543-3545 } \\
\text { Rv3504-3505 } \\
\text { Rv1094 } \\
\text { Rv0244 }\end{array}$ & $\begin{array}{l}\text { FadE28, FadE29 } \\
\text { FadE26, FadE27 } \\
\text { DesA2 } \\
\text { FadE5 }\end{array}$ \\
\hline Rv2175c & $\begin{array}{l}\text { Rv3550-3552 } \\
\text { Rv0469 }\end{array}$ & $\begin{array}{l}\text { EchA20 } \\
\text { UmA1 }\end{array}$ \\
\hline Rv2710(SigB) & $\begin{array}{l}\text { Rv0467-0468 } \\
\text { Rv2590 }\end{array}$ & $\begin{array}{l}\text { Icl, FadB2 } \\
\text { FadD9 }\end{array}$ \\
\hline Rv2745c & Rv2930-2939 & FadD26 \\
\hline Rv3133c(DevR) & $\begin{array}{l}\text { Rv0467-0468 } \\
\text { Rv2590 } \\
\text { Rv3504-3505 }\end{array}$ & $\begin{array}{l}\text { Icl, FadB2 } \\
\text { FadD9 } \\
\text { FadE26, FadE27 }\end{array}$ \\
\hline Rv3164c(MoxR3) & Rv2590 & FadD9 \\
\hline Rv3328 & Rv1094 & DesA2 \\
\hline Rv3334 & Rv3550-3552 & EchA20 \\
\hline Rv3414c(SigD) & Rv3537-3538 & UfaA2 \\
\hline Rv3416(WhiB3) & $\begin{array}{l}\text { Rv0244 } \\
\text { Rv0467-0468 } \\
\text { Rv0469 } \\
\text { Rv0551 } \\
\text { Rv1094 } \\
\text { Rv2930-2939 } \\
\text { Rv3504-3505 } \\
\text { Rv3537-3538 } \\
\text { Rv3543-3545 } \\
\text { Rv3550-3552 }\end{array}$ & $\begin{array}{l}\text { FadE5 } \\
\text { Icl, FadB2 } \\
\text { UmA1 } \\
\text { Fad8 } \\
\text { DesA2 } \\
\text { FadD26 } \\
\text { FadE26, FadE27 } \\
\text { UfaA2 } \\
\text { FadE28, FadE29 } \\
\text { EchA20 }\end{array}$ \\
\hline Rv3557c & Rv1094 & DesA2 \\
\hline Rv3678c & $\begin{array}{l}\text { Rv2590 } \\
\text { Rv0551 }\end{array}$ & $\begin{array}{l}\text { FadD9 } \\
\text { Fad8 }\end{array}$ \\
\hline Rv3692(MoxR2) & $\begin{array}{l}\text { Rv1094 } \\
\text { Rv2930-2939 }\end{array}$ & $\begin{array}{l}\text { DesA2 } \\
\text { FadD26 }\end{array}$ \\
\hline Rv3744 & Rv0244 & FadE5 \\
\hline Rv3833 & Rv0469 & UmA1 \\
\hline
\end{tabular}

The persistence of infection by M. tuberculosis is the result of a complex interplay between the host and the pathogen. However, little is known about the specific bacterial and host components involved in this process. An important role of transcriptional regulation in the life cycle of $M$. tuberculosis is suggested by genes encoding $\sim 150$ known or putative transcription regulatory proteins in the genome (Cole et al. 1998); however, their specific target genes have not been identified for most of these transcriptional factors. Several groups have suggested a role for a hypoxia- induced regulon (Dos) in the shift of M. tuberculosis from the replicative state to the persistent state (McKinney et al. 2000; Talaat et al. 2004; Rengarajan et al. 2005; Kumar et al. 2007; Rohde et al. 2007). In our study, oxygen stress regulators such as OxyS, DevR, and WhB3 were detected to bind directly with the regulating sequence of fatty acid metabolism or stress response genes, both of which form part of the core of in vivo-induced genes within the phagosome. Moreover, many putative transcription factors were found to participate in the regulation of both genes' expression. A further detailed study on these regulatory factors will provide important clues to understand better the mechanism of $M$. tuberculosis' adaptive response to extracellular redox signals and carbon source utilization.

Several previous studies support the hypothesis that M. tuberculosis switches its metabolism and utilizes fatty acid substrates as key nutrients in late-stage infected mice (McKinney et al. 2000; Timm et al. 2003). The metabolism of these substrates depends on carbon anaplerosis, an enzyme-catalyzed reaction that replenishes the intermediates in the cyclic pathway, through the glyoxylate shunt (McKinney et al. 2000; Muñoz-Elías and McKinney 2005). It has been suggested that an ordered metabolic switchover is also necessary for the adaptation of M. tuberculosis to anoxia (Singh et al. 2007). However, the specific factors involved in M. tuberculosis metabolism and environmental adaptation in vivo are largely unknown (Timm et al. 2003). H37Rv WhiB3 is homologous to the two Streptomyces proteins WhiD and WhiB3 (Molle et al. 2000). The deletion mutants of $w h i B$ and whiD were shown to critically influence spore development in S. coelicolor (Molle et al. 2000). This suggests that WhiB3 may be involved in sensing physiological changes. On the other hand, Singh et al. (2007) also observed the severe growth impairment of $M$. tuberculosis $\Delta$ whiB3 in various carbohydrates, and the mutant grew better on acetate as its sole carbon source. Based on the redox-sensing properties of WhiB3, together with the growth properties of its mutant on carbon-limiting media, WhiB3 has been suggested to play important roles in the regulation of both metabolic and redox switchover events (Singh et al. 2007). Thus, the proteins of the WhiB family, which can be induced by several stressful in vivo stimuli (Morris et al. 2005; Banaiee et al. 2006; Geiman et al. 2006), may facilitate an integrated transcriptional response to multiple stresses encountered within the phagosome. Indeed, the M. bovis whiB3 mutant was completely attenuated for growth in guinea pigs (Steyn et al. 2002), although the H37Rv whiB3 gene was not essential for growth in mouse and guinea pig models.

In this study, with the use of a new bacterial one-hybrid reporter system, we screened transcription factors for binding with regulatory sequences of fatty acid metabolism and stress response genes, both of which are part of the core of in vivo-regulated genes within the phagosome (McKinney et al. 2000; Talaat et al. 2004; Rengarajan et al. 2005; Rohde et al. 2007). Singh et al. (2007) have suggested that WhiB3 might play important roles in metabolic regulation and in sensing intracellular redox changes. Our current study found that WhiB3 could bind directly with the promoter sequences of most in vivo-induced genes (Tables 1,2). Previous findings have shown that WhiB3 binds with the major sigma factor RpoV and can be induced by several stressful in vitro stimuli (Steyn et al. 2002; Geiman et al. 2006). Therefore, our findings, together with those from previous reports, support a model in which M. tuberculosis WhiB3 is a core regulator for both metabolic switching and intracellular redox sensing. As such, it may facilitate integrated transcriptional responses to multiple stresses encountered within the phagosome. The specific recognition motif of

\section{Genome Research www.genome.org}


WhiB3 has not been characterized yet. In this study, we used an SPR assay and mapped a 60-bp potential target sequence for WhiB3 within the regulatory region of the icl gene cluster. However, when a further BLAST analysis was used to search the exact site, only nonconserved motifs could be found within the regulatory sequences of the target genes detected by the bacterial one-hybrid system (Supplemental Fig. 4C). Therefore, it was possible that WhiB3 simply had a relatively flexible DNA-binding specificity, which might make it important as a unique regulator participating in more extensive biological processes when $M$. tuberculosis invades the macrophages. This remains to be determined in future work.

In conclusion, we have developed a new bacterial one-hybrid system that provides a convenient and rapid technique for determining specific interactions between target DNA and transcription factors. About 100 previously unknown interactions of regulators with specific promoters were detected for modulations of the in vivo-induced genes' expression within the phagosome, including those involved in fatty acid metabolism and stress response genes (Supplemental Table 3). Some interactions have also been further confirmed by the SPR assay (Supplemental Table 4) or traditional EMSA techniques (Supplemental Fig. 5). However, although several previous in vivo studies confirmed some of the regulators' interactions with the DNA characterized in this study (Raman et al. 2004), the present data are all in vitro derived data, and thus most of the findings need to be confirmed directly in future work on M. tuberculosis. Nevertheless, important clues to further pursue the mechanisms on the adaptive responses and resistances of $M$. tuberculosis have been uncovered. Meanwhile, these proteins make attractive new drug targets because they may have essential roles in controlling the in vivo growth of $M$. tuberculosis in human hosts. Taken together, our results reveal important information for examining further the transcription regulation in $M$. tuberculosis. Moreover, the $\mathrm{B} 1 \mathrm{H}$ strategy presented here should be applicable to the study of DNA-binding factors in a wide range of biological organisms.

\section{Methods}

Bacterial strains, plasmids, enzymes, primers, and chemicals

Escherichia coli BL21 cells, purchased from Novagen, were used as the host strain to express M. tuberculosis proteins. pBT and pTRG vectors and $E$. coli host strains were purchased from Stratagene. pET28a was purchased from Novagen. The E. coli DH5 $\alpha$ host strain for cloning was purchased from Stratagene. Restriction enzymes, T4 ligase, and modification enzymes were from TaKaRa Biotech. Pyrobest DNA polymerase and deoxynucleoside triphosphates (dNTPs) were purchased from TaKaRa Biotech. All antibiotics were purchased from TaKaRa Biotech. All reagents for one-hybrid assay were purchased from Stratagene. All PCR primers were synthesized by Invitrogen (Supplemental Tables 1, 2). DNA purification kits were purchased from Watson Biotechnologies. $\mathrm{Ni}-\mathrm{NTA}\left(\mathrm{Ni}^{2+}\right.$-nitrilotriacetate) agarose columns were obtained from Qiagen.

\section{Construction of the bacterial one-hybrid reporter T-vector}

For developing a new bacterial one-hybrid (B1H) reporter vector, an $\sim 1.7-\mathrm{kb}$ DNA fragment containing the HIS3-aadA reporter cassette was cloned from the BacterioMatch II two-hybrid reporter system (Stratagene) using a pair of unique primers (Supplemental Table 1). This reporter cassette was further used to replace $\lambda c I$ and lac-UV5 promoters from $\mathrm{pBT}$ to produce a preliminary vector named pRpb (Supplemental Fig. 1). To restrain the self-activation and reduce screening background, a mediator DNA fragment was screened successfully from the genomic library of archaeon Sulfolobus solfataricus (data not shown) and the fragment was inserted into the right upstream of the HIS3-aadA reporter cassette. After further modifications, a derivative reporter vector of pRpb-S7 was produced, in which a BamHI restriction enzyme site was integrated into the upstream of the HIS3-aadA reporter cassette (Supplemental Fig. 1). For the convenient and rapid cloning of the short promoter DNA fragment into the one-hybrid system, we further engineered a 1.2-kb MCM $\Delta$ segment containing two XcmI sites in both its termini, the $\mathrm{XcmI}$ cassette. The cassette was derived from the minichromosome maintainance gene (MCM) of the archaeon $S$. solfataricus which was amplified by using a pair of specific primers containing the $\mathrm{XcmI}$ site (Supplemental Table 1). The XcmI cassette was inserted into BamHI-digested pRpb-S7 to produce a plasmid pBXcmT (Supplemental Fig. 1). When digested with XcmI, the recombinant plasmid $\mathrm{pBXcmT}$ resulted in a vector with a single deoxythymidine (dT) overhanging at its 3 '-end. This linearized T-vector could then be used for the rapid cloning of PCR products.

\section{Cloning of M. tuberculosis transcription factors and regulatory} sequences of the target genes and bacterial one-hybrid assays

About 150 transcription factors were predicted from the genome of M. tuberculosis (Cole et al. 1998). All of these probable transcription regulatory genes were amplified using their specific primers (Supplemental Table 5) and were cloned into the pTRG vector. A subgenomic library for $M$. tuberculosis transcription factors was produced by mixing these recombinant plasmids. The promoters of the $M$. tuberculosis genes were amplified using their specific primers (Supplemental Table 2) and were inserted directly into the XcmI site of pBXcmT. The E. coli XL1-Blue MRF' Kan strain was used for the routine propagation of all $\mathrm{pBXcmT}$ and $\mathrm{pTRG}$ recombinant plasmids. A pair of $\mathrm{pBXcmT/pTRG} \mathrm{plasmids} \mathrm{was} \mathrm{co-transformed} \mathrm{into}$ the reporter strain and its growth was then tested, together with the self-activation control on the selective medium containing 3-AT, $\mathrm{Kan}^{\mathrm{r}}$, Str ${ }^{\mathrm{r}}$, and $\mathrm{Chl}^{\mathrm{r}}$. To explore new regulators, the recombinant plasmid $\mathrm{pBXcmT}$ can also be used to screen the library for $M$. $t u$ berculosis transcription factors. Positive growth cotransformants were selected on a selective screening medium plate containing $20 \mathrm{mM}$ 3-AT, $16 \mu \mathrm{g} / \mathrm{mL}$ streptomycin, $15 \mu \mathrm{g} / \mathrm{mL}$ tetracycline, 34 $\mu \mathrm{g} / \mathrm{mL}$ chloramphenicol, and $50 \mu \mathrm{g} / \mathrm{mL}$ kanamycin. The plates were incubated at $30^{\circ} \mathrm{C}$ for $3-4 \mathrm{~d}$.

\section{Expression and purification of recombinant proteins of M. tuberculosis genes}

M. tuberculosis genes and their mutants were amplified by specific primers (Supplemental Table 1) from genomic DNA. The transcription factor genes were cloned into the overexpression vectors of pET28a to produce corresponding recombinant vectors. E. coli BL21-CodonPlus (DE3)-RIL cells transformed with the recombinant plasmid, were grown at $37^{\circ} \mathrm{C}$ in $1 \mathrm{~L}$ of $\mathrm{LB}$ medium containing $30 \mu \mathrm{g} / \mathrm{mL}$ chloramphenicol and $100 \mu \mathrm{g} / \mathrm{mL}$ ampicillin. Protein purification was carried out as described previously (He et al. 2003). Protein concentrations were determined according to a previously published procedure (He et al. 2003).

\section{Surface plasmon resonance analysis of protein-DNA interaction}

The interaction between the promoter DNA and the transcription factors was also assayed by surface plasmon resonance (SPR) using the BIAcore 3000 system. Biotin labeled promoter DNA was immobilized on the SA chips (BIAcore). Following a period of 
stabilization, the purified transcription regulatory protein was passed over the chip. Experiments were performed in a running buffer consisting of $100 \mathrm{mM}$ HEPES (pH 7.5), $50 \mu \mathrm{M}$ EDTA, 0.1 $\mathrm{mM}$ DTT, and $100 \mathrm{mM} \mathrm{NaCl}$ at a flow rate of $10 \mu \mathrm{L}$ per min at $25^{\circ} \mathrm{C}$. Each analysis was performed in triplicate. An overlay plot was produced for depicting the interaction between the transcription factor and the different concentrations of the promoter DNA; representative data are shown in the figures (Figs. 2D, 3B).

\section{Acknowledgments}

This work was supported by 973 Program (2006CB504402), the National Natural Science Foundation of China (30670016) and the National Special Key Project of China on Major Infectious Diseases (2008-2010).

\section{References}

Banaiee N, Jacobs WR Jr, Ernst JD. 2006. Regulation of Mycobacterium tuberculosis whiB3 in the mouse lung and macrophages. Infect Immun 74: $6449-6457$.

Berman BP, Nibu Y, Pfeiffer BD, Tomancak P, Celniker SE, Levine M, Rubin GM, Eisen MB. 2002. Exploiting transcription factor binding site clustering to identify cis-regulatory modules involved in pattern formation in the Drosophila genome. Proc Natl Acad Sci 99: 757-762.

Boon C, Dick T. 2002. Mycobacterium bovis (BCG) response regulator essential for hypoxic dormancy. J Bacteriol 184: 6760-6767.

Bulyk ML. 2005. Discovering DNA regulatory elements with bacteria. Nat Biotechnol 23: 942-944.

Champion PA, Stanley SA, Champion MM, Brown EJ, Cox JS. 2006. Cterminal signal sequence promotes virulence factor secretion in Mycobacterium tuberculosis. Science 313: 1632-1636.

Chen P, Ruiz RE, Li Q, Silver RF, Bishai WR. 2000. Construction and characterization of a Mycobacterium tuberculosis mutant lacking the alternate sigma factor gene, sigF. Infect Immun 68: 5575-5580.

Cole ST, Brosch R, Parkhill J, Garnier T, Churcher C, Harris D, Gordon SV, Eiglmeier K, Gas S, Barry CE III, et al. 1998. Deciphering the biology of Mycobacterium tuberculosis from the complete genome sequence. Nature 393: $537-544$

Derrick SC, Morris SL. 2007. The ESAT6 protein of Mycobacterium tuberculosis induces apoptosis of macrophages by activating caspase expression. Cell Microbiol 9: 1547-1555.

Florczyk MA, McCue LA, Stack RF, Hauer CR, McDonough KA. 2001. Identification and characterization of mycobacterial proteins differentially expressed under standing and shaking culture conditions, including Rv2623 from a novel class of putative ATP-binding proteins. Infect Immun 69: 5777-5785.

Geiman DE, Raghunand TR, Agarwal N, Bishai WR. 2006. Differential gene expression in response to exposure to antimycobacterial agents and other stress conditions among seven Mycobacterium tuberculosis whiBlike genes. Antimicrob Agents Chemother 50: 2836-2841.

Graham JE, Clark-Curtiss JE. 1999. Identification of Mycobacterium tuberculosis RNAs synthesized in response to phagocytosis by human macrophages by selective capture of transcribed sequences (SCOTS). Proc Natl Acad Sci 96: 11554-11559.

He ZG, Rezende LF, Willcox S, Griffith JD, Richardson CC. 2003. The carboxyl-terminal domain of bacteriophage T7 single-stranded DNAbinding protein modulates DNA binding and interaction with T7 DNA polymerase. J Biol Chem 278: 29538-29545.

James PE, Grinberg OY, Michaels G, Swartz HM. 1995. Intraphagosomal oxygen in stimulated macrophages. J Cell Physiol 163: 241-247.

Kumar A, Toledo JC, Patel RP, Lancaster JR Jr, Steyn AJ. 2007. Mycobacterium tuberculosis DosS is a redox sensor and DosT is a hypoxia sensor. Proc Natl Acad Sci 104: 11568-11573.

Lee BY, Hefta SA, Brennan PJ. 1992. Characterization of the major membrane protein of virulent Mycobacterium tuberculosis. Infect Immun 60: $2066-2074$.

Lee TI, Rinaldi NJ, Robert F, Odom DT, Bar-Joseph Z, Gerber GK, Hannett NM, Harbison CT, Thompson CM, Simon I, et al. 2002. Transcriptiona regulatory networks in Saccharomyces cerevisiae. Science 298: 799-804.

Liu X, Noll DM, Lieb JD, Clarke ND. 2005. DIP-chip: Rapid and accurate determination of DNA-binding specificity. Genome Res 15: 421-427.

Manganelli R, Voskuil MI, Schoolnik GK, Smith I. 2001. The Mycobacterium tuberculosis ECF sigma factor $\sigma^{\mathrm{E}}$ : Role in global gene expression and survival in macrophages. Mol Microbiol 41: 423-437.

McKinney JD, Höner zu Bentrup K, Muñoz-Elías EJ, Miczak A, Chen B, Chan WT, Swenson D, Sacchettini JC, Jacobs WR Jr, Russell DG. 2000
Persistence of Mycobacterium tuberculosis in macrophages and mice requires the glyoxylate shunt enzyme isocitrate lyase. Nature $\mathbf{4 0 6}$ 735-738.

Meng X, Brodsky MH, Wolfe SA. 2005. A bacterial one-hybrid system for determining the DNA-binding specificity of transcription factors. Nat Biotechnol 23: 988-994.

Molle V, Palframan WJ, Findlay KC, Buttner MJ. 2000. WhiD and WhiB, homologous proteins required for different stages of sporulation in Streptomyces coelicolor A3(2). J Bacteriol 182: 1286-1295.

Morris RP, Nguyen L, Gatfield J, Visconti K, Nguyen K, Schnappinger D, Ehrt S, Liu Y, Heifets L, Pieters J, et al. 2005. Ancestral antibiotic resistance in Mycobacterium tuberculosis. Proc Natl Acad Sci 102: 12200-12205.

Muñoz-Elías EJ, McKinney JD. 2005. Mycobacterium tuberculosis isocitrate lyases 1 and 2 are jointly required for in vivo growth and virulence. Nat Med 11: 638-644.

Parish T, Smith DA, Roberts G, Betts J, Stoker NG. 2003. The senX3-regX3 two-component regulatory system of Mycobacterium tuberculosis is required for virulence. Microbiology 149: 1423-1435.

Park HD, Guinn KM, Harrell MI, Liao R, Voskuil MI, Tompa M, Schoolnik GK, Sherman DR. 2003. Rv3133c/dosR is a transcription factor that mediates the hypoxic response of Mycobacterium tuberculosis. Mol Microbiol 48: 833-843.

Raman S, Hazra R, Dascher CC, Husson RN. 2004. Transcription regulation by the Mycobacterium tuberculosis alternative sigma factor SigD and its role in virulence. J Bacteriol 186: 6605-6616.

Rengarajan J, Bloom BR, Rubin EJ. 2005. Genome-wide requirements for Mycobacterium tuberculosis adaptation and survival in macrophages. Proc Natl Acad Sci 102: 8327-8332.

Rohde KH, Abramovitch RB, Russell DG. 2007. Mycobacterium tuberculosis invasion of macrophages: Linking bacterial gene expression to environmental cues. Cell Host Microbe 2: 352-364.

Roulet E, Busso S, Camargo AA, Simpson AJ, Mermod N, Bucher P. 2002. High-throughput SELEX-SAGE method for quantitative modeling of transcription-factor binding sites. Nat Biotechnol 20: 831-835.

Sassetti CM, Rubin EJ. 2003. Genetic requirements for mycobacterial survival during infection. Proc Natl Acad Sci 100: 12989-12994.

Schaible UE, Sturgill-Koszycki S, Schlesinger PH, Russell DG. 1998. Cytokine activation leads to acidification and increases maturation of Mycobacterium avium-containing phagosomes in murine macrophages. J Immunol 160: 1290-1296.

Sherman DR, Voskuil M, Schnappinger D, Liao R, Harrell MI, Schoolnik GK 2001. Regulation of the Mycobacterium tuberculosis hypoxic response gene encoding $\alpha$-crystallin. Proc Natl Acad Sci 98: 7534-7539.

Singh A, Guidry L, Narasimhulu KV, Mai D, Trombley J, Redding KE, Giles GI, Lancaster JR Jr, Steyn AJ. 2007. Mycobacterium tuberculosis WhiB3 responds to $\mathrm{O}_{2}$ and nitric oxide via its [4Fe-4S] cluster and is essential for nutrient starvation survival. Proc Natl Acad Sci 104: 11562-11567.

Steyn AJ, Collins DM, Hondalus MK, Jacobs WR Jr, Kawakami RP, Bloom BR. 2002. Mycobacterium tuberculosis WhiB3 interacts with RpoV to affect host survival but is dispensable for in vivo growth. Proc Natl Acad Sci 99: 3147-3152.

Talaat AM, Lyons R, Howard ST, Johnston SA. 2004. The temporal expression profile of Mycobacterium tuberculosis infection in mice. Proc Natl Acad Sci 101: 4602-4607.

Timm J, Post FA, Bekker LG, Walther GB, Wainwright HC, Manganelli R, Chan WT, Tsenova L, Gold B, Smith I, et al. 2003. Differential expression of iron-, carbon-, and oxygen-responsive mycobacterial genes in the lungs of chronically infected mice and tuberculosis patients. Proc Natl Acad Sci 100: 14321-14326.

Verbon A, Hartskeerl RA, Schuitema A, Kolk AH, Young DB, Lathigra R. 1992. The 14,000-molecular-weight antigen of Mycobacterium tuberculosis is related to the alpha-crystallin family of low-molecularweight heat shock proteins. J Bacteriol 174: 1352-1359.

Via LE, Fratti RA, McFalone M, Pagan-Ramos E, Deretic D, Deretic V. 1998 Effects of cytokines on mycobacterial phagosome maturation. J. Cell Sci. 111: 897-905.

Wayne LG, Sohaskey CD. 2001. Nonreplicating persistence of Mycobacterium tuberculosis. Annu Rev Microbiol 55: 139-163.

Wilkinson RJ, DesJardin LE, Islam N, Gibson BM, Kanost RA, Wilkinson KA, Poelman D, Eisenach KD, Toossi Z. 2001. An increase in expression of a Mycobacterium tuberculosis mycolyl transferase gene ( $f b p B)$ occurs early after infection of human monocytes. Mol Microbiol 39: 813-821.

Yuan Y, Crane DD, Simpson RM, Zhu YQ, Hickey MJ, Sherman DR, Barry CE III. 1998. The $16-\mathrm{kDa} \alpha$-crystallin (Acr) protein of Mycobacterium tuberculosis is required for growth in macrophages. Proc Natl Acad Sci 95: 9578-9583.

Zahrt TC, Deretic V. 2000. An essential two-component signal transduction system in Mycobacterium tuberculosis. J Bacteriol 182: 3832-3838.

Received September 15, 2008; accepted in revised form January 9, 2009.

1308 Genome Research

www.genome.org 
Genome Research 19: 1301-1308 (2009)

Dissecting transcription regulatory pathways through a new bacterial one-hybrid reporter system

Manman Guo, Hui Feng, Jun Zhang, Wenqin Wang, Yi Wang, Yuqing Li, Chunhui Gao, Huanchun Chen, Ying Feng, and Zheng-Guo He

The e-mail address for the corresponding author provided in this paper is no longer functional and has been changed to he.zhengguo@hotmail.com. 


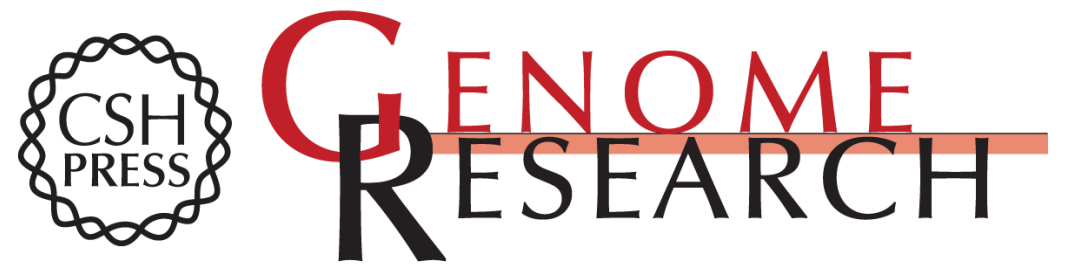

\section{Dissecting transcription regulatory pathways through a new bacterial one-hybrid reporter system}

Manman Guo, Hui Feng, Jun Zhang, et al.

Genome Res. 2009 19: 1301-1308 originally published online February 18, 2009

Access the most recent version at doi:10.1101/gr.086595.108

\section{Supplemental Material \\ Related Content}

References

This article cites 42 articles, 28 of which can be accessed free at:
http://genome.cshlp.org/content/19/7/1301.full.html\#ref-list-1

Articles cited in:

http://genome.cshlp.org/content/19/7/1301.full.html\#related-urls

\section{Erratum}

Genome Res. September , 2009 19: 1691

\section{License} \\ Email Alerting $\begin{aligned} & \text { Receive free email alerts when new articles cite this article - sign up in the box at the } \\ & \text { Service }\end{aligned}$ top right corner of the article or click here.
}

http://genome.cshlp.org/content/suppl/2009/02/20/gr.086595.108.DC1

\section{Affordable, Accurate Sequencing.}

(a) 